\title{
Multimodal Medical Image Fusion Based On SVD
}

\author{
P.Ambika Priyadharsini ${ }^{1}$, M.R.Mahalakshmi ${ }^{2}$ \\ I'Applied Electronics, Sri Muthukumaran Institute of Technology, Chennai, India) \\ ${ }^{2}$ (Assistant Proffesor Department of ECE, Sri Muthukumaran Institute of Technology, Chennai, India)
}

\begin{abstract}
Image fusion is a promising process in the field of medical image processing, the idea behind is to improve the content of medical image by combining two or more multimodal medical images. In this paper a novel fusion framework based on singular value decomposition - based image fusion algorithm is proposed. $S V D$ is an image adaptive transform, it transforms the matrix of the given image into product USV ${ }^{T}$, which allows to refactor a digital image into three matrices called tensors. The proposed algorithm picks out informative image patches of source images to constitute the fused image by processing the divided subtensors rather than the whole tensor and a novel sigmoid-function-like coefficient-combining scheme is applied to construct the fused result. Experimental results show that the proposed algorithm is an alternative image fusion approach.
\end{abstract}

Keywords: Coefficient-combining strategy, image fusion, sigmoid function, singular value decomposition

\section{INTRODUCTION}

Fusion (also called synthesis) is the process of combining two or more distinct entities into a new whole. Image fusion is the process of combining relevant information from two or more images into a single image. The resulting image will be more informative than any of the input images. With rapid advancements in technology, it is now possible to obtain information from multi source images to produce a high quality fused image with spatial and spectral information. Image Fusion is a mechanism to improve the quality of information from a set of images. Important applications of the fusion of images include medical imaging, microscopic imaging, remote sensing, computer vision, and robotics. Use of the Simple primitive technique will not recover good fused image in terms of performance parameter like peak signal to noise ratio (PSNR), Normalized correlation (NC), and Mean square error (MSE). Recently Discrete Wavelet Transform (DWT) and Principal Component Analysis (PCA) techniques have been popular fusion techniques in image processing.These methods are shown to perform much better than simple averaging max and min. The purpose of this paper is to demonstrate an image enhancement technique for easy, rapid \& effective mapping of investigating areas, separating the needed spectral information into one new component. Resultant image quality is superior to any of the input images.

\section{EXISTING IMAGE FUSION TECHNIQUES}

Image fusion method can be broadly classified into two groups

1. Spatial domain fusion method.

2. Transform domain fusion.

In spatial domain techniques, we directly deal with the image pixels. The pixel values are manipulated to achieve desired result. The fusion methods such as simple average method and principal component analysis (PCA) based methods fall under spatial domain approaches. The disadvantage of spatial domain approaches is that they produce spatial distortion in the fused image. In transform domain method image is first transferred into frequency domain. It means that the Fourier Transform of the image is computed first. All the Fusion operations are performed on the Fourier transform of the image and then the Inverse Fourier transform is performed to get the resultant image. The fusion method such as DWT and SVD fall under transform domain method.

\subsection{Simple Average Method}

In this method the resultant fused image is obtained by taking the average intensity of corresponding pixels from both the input image. 


\subsection{Principal Component Analysis (PCA) method}

Principal Component Analysis is a sub space method, which reduces the multidimensional data sets into lower dimensions for analysis. This method determines the weights for each source. image using the eigenvector corresponding to the largest eigen value of the covariance matrix of each source image.

\subsection{Discrete Wavelet Transform Method}

Wavelet transforms are multi-resolution image decomposition tool that provide a variety of channels representing the image feature by different frequency subbands at multi-scale. It is a famous technique in analyzing signals. When decomposition is performed, the approximation and detail component can be separated 2-D Discrete Wavelet Transformation (DWT) converts the image from the spatial domain to frequency domain. The image is divided by vertical and horizontal lines and represents the first-order of DWT, and the image can be separated with four parts those are LL1, LH1, HL1 and HH1. Applying Inverse Discrete Wavelet Transform on fused decomposed level, reconstruct the fused output image.

\section{IMAGE FUSION TECHNIQUE USING SVD}

In this paper, a novel Image fusion technique based on Higher Order singular value decomposition (HOSVD) is presented to fuse the source images. SVD is a method for identifying and ordering the dimensions along which data points exhibit the most variations. Using SVD it's possible to find the best approximation of the original data points using fewer dimensions. SVD takes a high dimensional, highly variable set of data points and reducing it to a lower dimensional space that exposes the substructure of the original data more clearly and orders it from most variation to the least. SVD is based on a theorem from linear algebra which says that a rectangular matrix A can be broken down into the product of three matrices - an orthogonal matrix $\mathrm{U}$, a diagonal matrix S, and the transpose of an orthogonal matrix $\mathrm{V}$. The theorem is usually presented like this:

$$
\mathrm{A}=\mathrm{USV}^{\mathrm{T}}
$$

where $\mathrm{UTU}=\mathrm{I}, \mathrm{VTV}=\mathrm{I}$; the columns of $\mathrm{U}$ are orthonormal eigenvectors of $\mathrm{AA}^{\mathrm{T}}$, the columns of $\mathrm{V}$ are orthonormal eigenvectors of $\mathrm{A}^{\mathrm{T}} \mathrm{A}$, and $\mathrm{S}$ is a diagonal matrix containing the square roots of eigenvalues from $\mathrm{U}$ or $\mathrm{V}$ in descending order. To compute the SVD of a matrix A with $m$ rows and $n$ columns, with rank $r$ and $r \leq n$ $\leq \mathrm{m}$. Then the A can be factorized into three matrices.

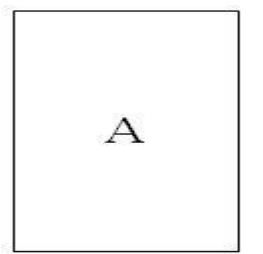

$m \times n$
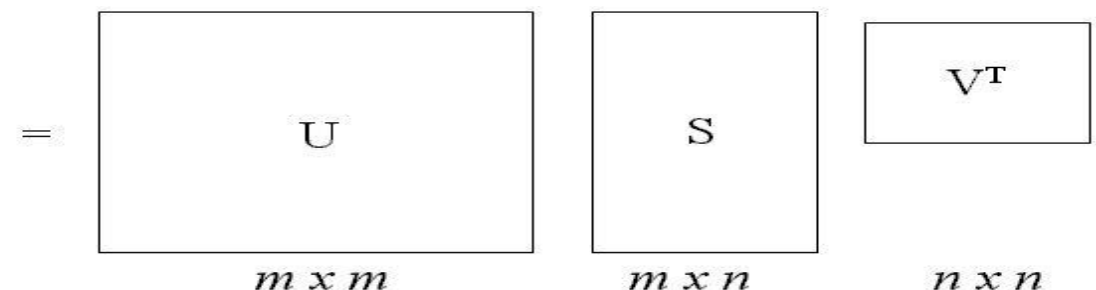

$n \times n$

Where $\mathrm{U}$ and $\mathrm{V}$ are orthonormal and $\mathrm{S}$ is diagonal. Matrix $\mathrm{U}$ is an $\mathrm{m} \times \mathrm{m}$ orthogonal matrix

$$
\mathrm{U}=\left[\mathrm{u}_{1}, \mathrm{u}_{2}, \ldots \mathrm{u}_{\mathrm{r}}, \mathrm{u}_{\mathrm{r}+1}, \ldots, \mathrm{u}_{\mathrm{m}}\right]
$$

column vectors $\mathrm{u}_{\mathrm{i}}$, for $\mathrm{i}=1,2, \ldots, \mathrm{m}$, form an orthonormal set:

And matrix $\mathrm{V}$ is an $\mathrm{n} \times \mathrm{n}$ orthogonal matrix

$$
\mathrm{V}=\left[\mathrm{v}_{1}, \mathrm{v}_{2}, \ldots \mathrm{v}_{\mathrm{r}}, \mathrm{v}_{\mathrm{r}+1}, \ldots, \mathrm{v}_{\mathrm{n}}\right]
$$

Column vectors $\mathrm{i} v$ for $\mathrm{i}=1,2, \ldots, \mathrm{n}$, form an orthonormal set: Here, $\mathrm{S}$ is an $\mathrm{m} \times \mathrm{n}$ diagonal matrix with singular values $(\mathrm{SV})$ on the diagonal. The matrix $S$ can be showed in following

$$
\mathrm{S} 12=\left[\begin{array}{cc}
\mathrm{S}_{1}(1)^{2} & 0 \\
0 & \mathrm{~S}_{2}(2)^{2}
\end{array}\right.
$$


It contains the squares of the singular values, with $s_{1}(1)>\underline{s_{2}(2)}$.

\section{Proposed Image Fusion Algorithm Using Svd}

SVD consider the source image as tensors, Tensors (multiway arrays) are generalizations of scalars, vectors, and matrices to an arbitrary number of indices. Tensor-based information processing methods are more suitable for representing high-dimensional data and extracting relevant information than vector- and matrix based methods. As one of most efficient tensor decomposition techniques, higher order singular value decomposition (HOSVD) based image fusion algorithm is proposed. It is worthwhile to highlight several aspects of the proposed transform domain-based approach here.

i) Since source images refer to the same scene and are somewhat similar (the same physical structures in the environment), this paper constructs them into a tensor and employs the HOSVD technique to extract their features simultaneously. Furthermore, because image fusion depends on local information of source images rather than total information, the proposed algorithm picks out informative image patches of source images to constitute the fused image by processing the divided subtensors rather than the whole tensor.

ii) A slice of the core tensor yielded from HOSVD of subtensors reflects the quality of the related image patch. This paper employs the sum of absolute values of coefficients (SAVC) as the activity-level measurement of the related patch.

iii) To adapt to different activity-level measurements, this paper proposes a novel and flexible sigmoidfunction-like coefficient-combining scheme, which incorporates the usual choose-max scheme and the weighted average scheme, and easily extends the proposed algorithm to fuse multiple or color images.

The input images refered in the below block diagram may be a multimodality medical images such as CT, MRI, PET etc.,

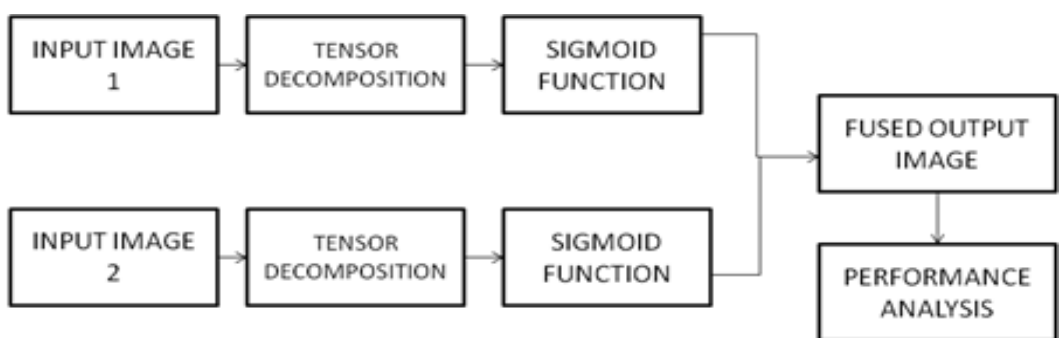

Fig.4 block diagram of image fusion using svd

\subsection{Description of the Proposed Algorithm}

Generally, a transform-domain fusion algorithm consists of the following three steps:

4.1.1 Obtain the decomposition coefficients using some transform

4.1.2 Construct the activity-level measurement from these coefficients and

4.1.3 Merge these coefficients to construct the fused result in line with the measurements above.

\subsection{Fusion rules schemes implemented using SVD}

4.2.1 Maximum selection (MS) scheme:

This simple scheme just picks the coefficient in each sub band with the largest magnitude.

4.2.2 Weighted average (WA) scheme:

This scheme developed by Burt and Kolczynski uses a normalized correlation between the two images sub bands over a small local area. The Resultant coefficient for reconstruction is calculated from this measure via a weighted average of the two images coefficients

\section{ReSUlts AND ANALYSIS}

To verify the proposed method, it is tested on medical images. The general requirement of an image fusing process is to preserve all valid and useful information from the source images, while at the same time it should not introduce any distortion in resultant fused image. 


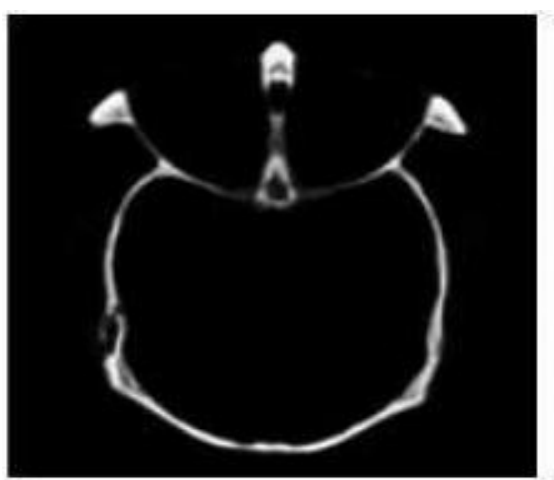

Fig 6.1 Source Images

a) CT image
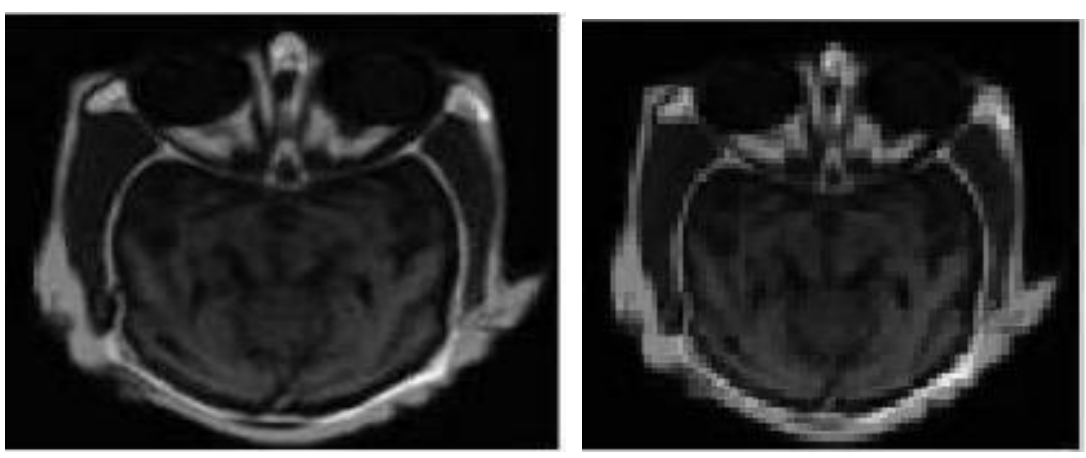

Fig 6.2 Fused Output

c) simple average method

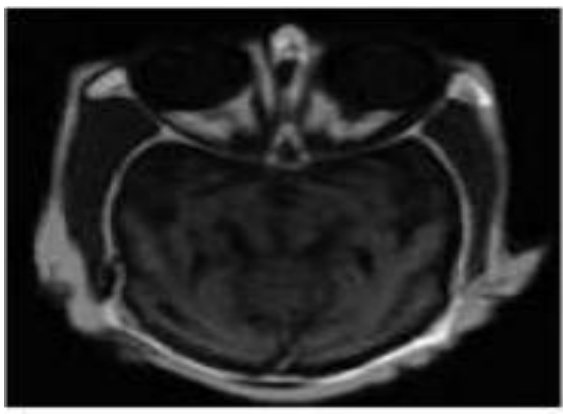

c) DWT

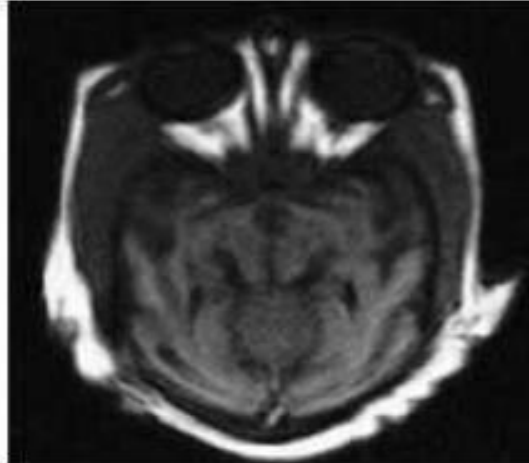

b) MRI image d) $\mathrm{PCA}$

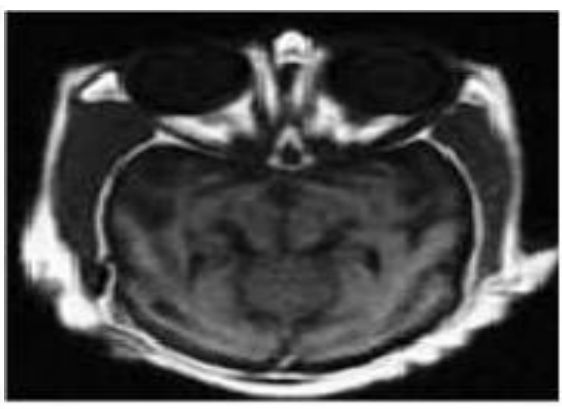

d) SVD

Performance measures are essential to measure the possible benefits of fusion and also used to compare results obtained with different algorithms.

\section{a. Peak Signal to Noise Ratio}

The PSNR is use to calculate the similarity between two images. The PSNR between the reference image $\mathrm{R}$ and the fused image $\mathrm{F}$ is defined as

$$
P S N R=10 \times \log _{10}\left(\frac{255}{R M S E}\right)
$$

For better fused image PSNR value should high.

\section{b. Normalized Cross Correlation}

The Normalized Cross Content between the reference image $\mathrm{R}$ and the fused image F is defined as

$$
N C C=\sum_{\mathrm{i}=1}^{\mathrm{m}} \sum_{\mathrm{j}=1}^{\mathrm{n}} \mathrm{R}_{\mathrm{ij}} * \mathrm{~F}_{\mathrm{ij}} \quad / \sum_{\mathrm{i}=1}^{\mathrm{m}} \sum_{\mathrm{j}=1}^{\mathrm{n}} \mathrm{R}^{2}
$$




\section{c. $\quad$ Entropy (EN)}

Entropy is used to calculate the amount of information. Higher value of entropy indicates that the information increases and the fusion performances are improved.

$$
E=\sum_{i=0}^{l-1} p_{i} \log _{2} p_{i}
$$

TABLE 1

Comparison of Different Image Fusion Techniques

\begin{tabular}{|c|c|c|c|c|c|}
\hline S.NO & $\begin{array}{c}\text { FUSION } \\
\text { TECHNIQUE }\end{array}$ & DOMAIN & $\begin{array}{c}\text { MEASURING } \\
\text { PARAMETER }\end{array}$ & ADVANTAGE & DISADVANTAGE \\
\hline 1 & $\begin{array}{c}\text { Simple } \\
\text { Average }\end{array}$ & Spatial & $\begin{array}{c}\text { PSNR=46.66 } \\
\text { NCC }=0.95 \\
\text { EN=7.4122 }\end{array}$ & $\begin{array}{c}\text { Simple to } \\
\text { implement }\end{array}$ & $\begin{array}{c}\text { High information } \\
\text { loss }\end{array}$ \\
\hline 2 & PCA & Spatial & $\begin{array}{c}\text { PSNR=66.66 } \\
\text { NCC }=0.96 \\
\text { EN=7.4128 }\end{array}$ & Simple algorithm & $\begin{array}{c}\text { Presence of } \\
\text { spectral } \\
\text { degradation }\end{array}$ \\
\hline 3 & DWT & Transform & $\begin{array}{c}\text { PSNR=66.66 } \\
\text { NCC }=0.97 \\
\text { EN=7.4244 }\end{array}$ & $\begin{array}{c}\text { Better Signal to } \\
\text { noise ratio }\end{array}$ & $\begin{array}{c}\text { Less spatial } \\
\text { Resolution }\end{array}$ \\
\hline 4 & SVD & Transform & $\begin{array}{c}\text { PSNR=70.44 } \\
\text { NCC }=0.98 \\
\text { EN=7.4216 }\end{array}$ & $\begin{array}{c}\text { Robust, simple, } \\
\text { fast to implement, } \\
\text { efficiency is more } \\
\text { even in the } \\
\text { presence of noise }\end{array}$ & $\begin{array}{c}\text { Efficiency is still } \\
\text { improved if noise is } \\
\text { reduced }\end{array}$ \\
\hline
\end{tabular}

\section{CONCLUSION}

Thus a novel image adaptive transform called SVD has been proposed. The proposed algorithm splits the multiple input images into tensor and can evaluate the quality of image patches using SVD of subtensors. Finally fused result is obtained by employing sigmoid function. Experimental results and performance analysis prove that the proposed algorithm is an alternative image fusion approach because it is simple, robust, fast to implement and it works well in constrained environment.

\section{REFERENCES}

[1] Junli Liang, Yang He, Ding Liu and Xianju Zeng (2012) 'Image Fusion Using Higher Order Singular Value Decomposition', IEEE Transactions On Image Processing, Volume 21, No.5, pp 2898-2909.

[2] Amarjot Kaur and Sunil Khullar (2013) 'Image Fusion using HIS, PCA and Wavelet Technique', International Journal of Computer Science and Communication Engineering, Volume 2, No. 2, pp. 92-94.

[3] V.P.S. Naidu (2011) 'Image Fusion Technique using Multi-resolution Singular Value Decomposition', Defence Science Journal, Volume 61, No. 5 pp. 479-484.

[4] G. Bergqvist and E.G Larsson (2010) 'The higher-order singular value decomposition Theory and application', IEEE Signal Processing Magazine, volume 27, No. 3, pp. 151-154.

[5] Li H., S. Manjunath and S. Mitra (1995) 'Multi sensor image fusion using the wavelet transform', International journal of Graphical Models and Image Processing, volume 57, No.3, pp. 235-245.

[6] Cyn Dwith., Vivek Angoth and Amarjoth Singh (2013) 'Wavelet Based Image Fusion for Detection of Brain Tumor', International Journal of Image, graphics and Signal Processing, Volume 1, pp.25-31.

[7] J. Canny (1986) 'A Computational Approach to Edge Detection', IEEE Transactions Pattern Analysis Intelligence Volume 8, pp. 679-714.

[8] K.L. Chung, C.H. Shen and L.C. Chang (2001) 'A novel SVD and VQ based image hiding scheme', International Journal of Advance Research Electronics and communication, Volume 22, pp. 105- 108.

[9] Daljit Kaur and Palwinder Singh Mann (2013) 'Hybrid Transform Domain Algorithm for Medical Image Fusion', International Journal for Science and Emerging Technologies with Latest Trends, Volume 8, No.1, pp. 23-27.

[10] R. Costantini, L. Sbaiz and S. Susstrunk (2008) 'Higher order SVD analysis for dynamic texture synthesis', IEEE Transactions on Image Processing, volume 17, No.1, pp.42-52

[11] Bibo Lu. Chunli Miao. (2010) 'Structure Tensor Based Image Fusion’, Proc.Third International Symposium on Electronic Commerce and Security Workshops, Volume 11, No.4, pp. 343-346.

[12] Aristeidis Sotiras., Christos Davatzikos and Nikos Paragios (2013) 'Deformable Medical Image Registration: A Survey', IEEE Transactions on Medical imaging, Volume 32, No. 7, pp. 1153-1190.

[13] Q.M. Gaurav Bhatnagar, JonathanWu and Zheng Liu 'Directive Contrast Based Multimodal Medical Image Fusion in NSCT Domain’ IEEE TRANSACTIONS ON MULTIMEDIA, VOLUME 15, NO. 5, AUGUST 2013 